\title{
VISUALIZATION AND ANALYSIS OF MOLECULAR POTENTIAL ENERGY SURFACE (PES) AND ITS MINIMA
}

\author{
Eugene Popov ${ }^{1}$, Anatoly Batiukov ${ }^{1}$, Natalja Vogt ${ }^{2}$, Tatyana Popova ${ }^{3}$ and Jürgen Vogt ${ }^{2}$ \\ ${ }^{I}$ Nizhegorodsky State Architectural \& Civil Engineering University, 65, Ilyinskaya Street, Nizhny Novgorod, \\ 603950, Russia \\ ${ }^{2}$ University of Ulm, Albert Einstein Allee 47, D-89069 Ulm, Germany \\ ${ }^{3}$ National Research University Higher School of Economics, 25/12, Bolshaja Pecherskaja Ulitsa, Nizhny Novgorod, \\ 603155, Russia
}

\begin{abstract}
The study of the potential energy surface (PES) of a molecule is of fundamental importance in structural chemistry. PES, being a function of torsion angles describing the internal rotation of different fragments in the molecule, is usually calculated by means of standard programs for quantum-chemical calculations (for instance, by GAUSSIAN). The supplementing program (GAUSSVIEW) suggests the visualization of PES with not more than two torsional coordinates (3D PES). The visualization of four-dimensional and more complex hypersurfaces is still a challenge for structural chemists. Another problem is finding all local minima of hypersurfaces corresponding to the conformational landscape of the molecule. This paper describes how a specially designed multidimensional postprocessor can be used to solve these problems. It also demonstrates the postprocessor capabilities by presenting a few examples.
\end{abstract}

\section{KEYWORDS}

Multidimensional Postprocessor, Visualization and Analysis of Multidimensional Function, Java, Java3D, Conformations of Molecules with Several Internal Rotors

\section{INTRODUCTION}

Understanding and predicting the properties of chemical compounds are of paramount importance both from technological and academic points of view. Quantum chemical computational methods become the useful tools for solving some chemical problems and studying the reaction mechanism prior to difficult, expensive or even impossible experiments.

There are several computer program packages applied to solve this problem, for example, GAUSSIAN, GAMESS, MOLPRO, SPARTAN, CFOUR, etc. The widely used program GAUSSIAN is supplemented by the visualization tool (GAUSSVIEW) which allows the graphical presentation of obtained results, for instance visualization of the potential energy surface (PES) of the molecule. As a function of torsion angles describing the internal rotation of the different fragments in the molecule, PES yields the information concerning the stable conformations of the molecule (conformers), corresponding to minima on PES, and the barrier height of interconversion between the conformers. However, the graphical presentation of PES is limited by two geometrical variables (3D PES). The visualization of four-dimensional and more complex hypersurfaces is still a challenge for structural chemists. Therefore, there is a need to create a reliable and affordable post-processor for visualization and minima finding of complex hypersurfaces. In the present work, we used the output of GAUSSIAN 03 program package (Frisch, 2003). 


\section{DISPLAING MULTIDIMENSIONAL PES}

The multidimensional postprocessor should carry out two main duties, namely: searching all the minima on multidimensional PES calculated by GAUSSIAN program and their visualization. The main requirements for visualization are the clarity of the surface and its behavior in the considered range, the ability to display all the minima found in its area, and the ability to observe the obtained results from any point of view. The PES calculations are usually associated with extremely high computer time. Therefore, they are usually made on a sparse grid. In the case of the function of 4 variables, the dimension of the grid cannot be made more than $6 \times 6 \times 6 \times 6$. In addition, the program leads to multidimensional tables that are extremely difficult to analyze in terms of finding energy minima. The only way to improve the situation is to increase the grid density at the postprocessing stage. We have developed a reliable and efficient algorithm for such increasing.

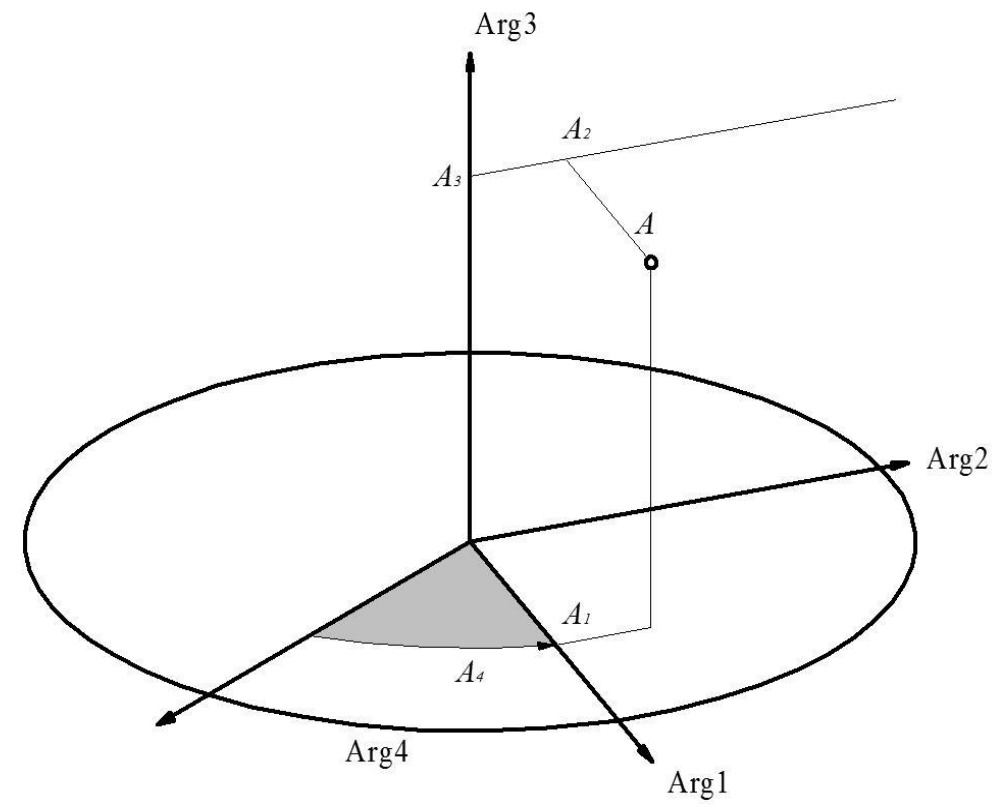

Figure 1. Special co-ordinate system to display 5D function (Four arguments + color)

We have developed a postprocessor that allows us to visualize and find the minima of 3D, 4D and 5D hypersurfaces. Visualization of the three-dimensional surface is carried out by the NURBS approximation and it is not difficult. To visualize 4D function we apply the voxel representation as introduced, for example, by James Foley (Foley et al, 1990). A much more complex problem is to visualize 5D function. Often, when analyzing multidimensional data, dimensionality reduction (DR) techniques are displayed in form of 2D or 3D scatterplots that project the multidimensional points onto a lower-dimensional visual space (Etemadpour $\mathrm{R}$. et al, 2016). One can also reconstruct 5D function to 4D function with energy level represented by color and apply the model called Lumigraph by S.Gotler (Gotler, 1996). The Lumigraph is a subset of the complete plenoptic function that describes the flow of light in all positions and in all directions. Unfortunately, though such representation is very simple and convenient it is poor for perception. An example of multidimensional visualization is the Self-Organizing Map (SOM). The SOM algorithm is a special type of neural network developed by Kohonen (2001) in the 1980s. The SOM algorithm creates a two-dimensional layout in which multidimensional data is presented, so that similar data elements are located close to each other. The SOM technique can be used to visualize large multidimensional data sets (by projecting data into low-dimensional space) and for clustering. 
There are many other methods to generate 2D similarity-based layouts from a higher-dimensional space. For example, the method of paired distances between points by I. Borg, et al (see Borg, (2010)) implemented with multidimensional scaling (MDS), taking into account the distances within the cluster or the distances between the clusters (Tenembaum, 2000). Sometimes Principal component analysis (PCA) is applied to generate similarity layouts by reducing data to lower dimensional visual spaces (Jollife, 1986). Some methods, such as isometric object mapping (Isomap), preferably use distances between data clusters. However, all of them are insufficient in our case for solving the problem of minima finding of the hypersurface and its visualization.

In our turn, we applied a special coordinate system to solve this problem (see Figure 1), which includes four independent variables. Such co-ordinate system makes point A in Figure 1 a five-dimensional point including its color. To visualize 5D function we introduce a set of 5D elementary hyper volumes (tetraxels) (Vogt, 2009).

To develop multidimensional postprocessor we choose Java language as a programming tool (Niemeyer, 2013) in conjunction with the Java 3D extension (Selman, 2003). The selected API provides a set of high-level tools for creating and manipulating three-dimensional geometry, as well as for pleasant rendering.

\section{MINIMA FINDING OF MULTIDIMENSION PES}

PES calculated and tabulated by GAUSSIAN has no analytic expression, which seriously complicates the search for its minima. Therefore, we developed the approach for PES local minima finding consisting of two stages:

1. We need to replace the initial multidimensional function defined as a regular grid of calculated points by more condensed regular grid. The function values at the nodes of condensed grid are calculated by multidimensional spline interpolation. In this version of the post-processor, we apply a recursive cubic spline interpolation procedure developed by Flanagan M. (2014) that allows interpolating the values of a function specified in a table of any dimension.

2. Further, we need to consistently compare the function values at each grid node with the function values at the neighboring nodes surrounding it. If this value is the smallest one, then we consider this value as a local minimum. The number of neighboring nodes depends on the dimension of the problem being solved. In 3D case, such nodes are 8 , in 4D case - 26, in 5D case, such nodes are 80 (See Figure 2). To make minima more accurate we should condense the grid as much as possible.

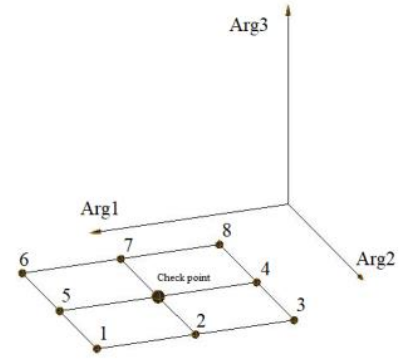

a)

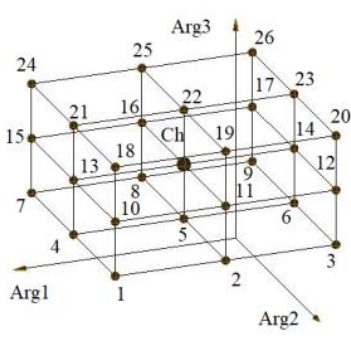

b)

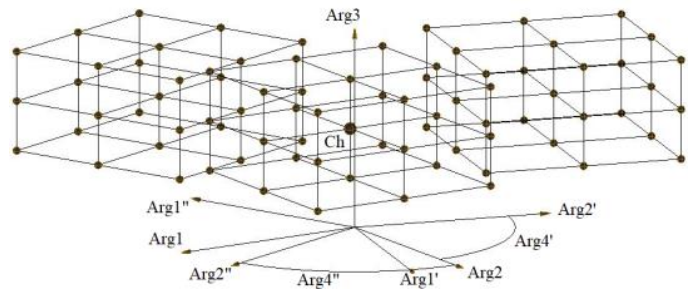

c)

Figure 2. The scheme of minima finding. a) $3 \mathrm{D}$ case; b) $3 \mathrm{D}+$ color $=4 \mathrm{D}$; c) $4 \mathrm{D}+$ color $=5 \mathrm{D}$

This approach allows the finding of energy function minima at the sufficient accuracy level. Figure 3 shows the first 11 found minima together with a 5D hypersurface. 


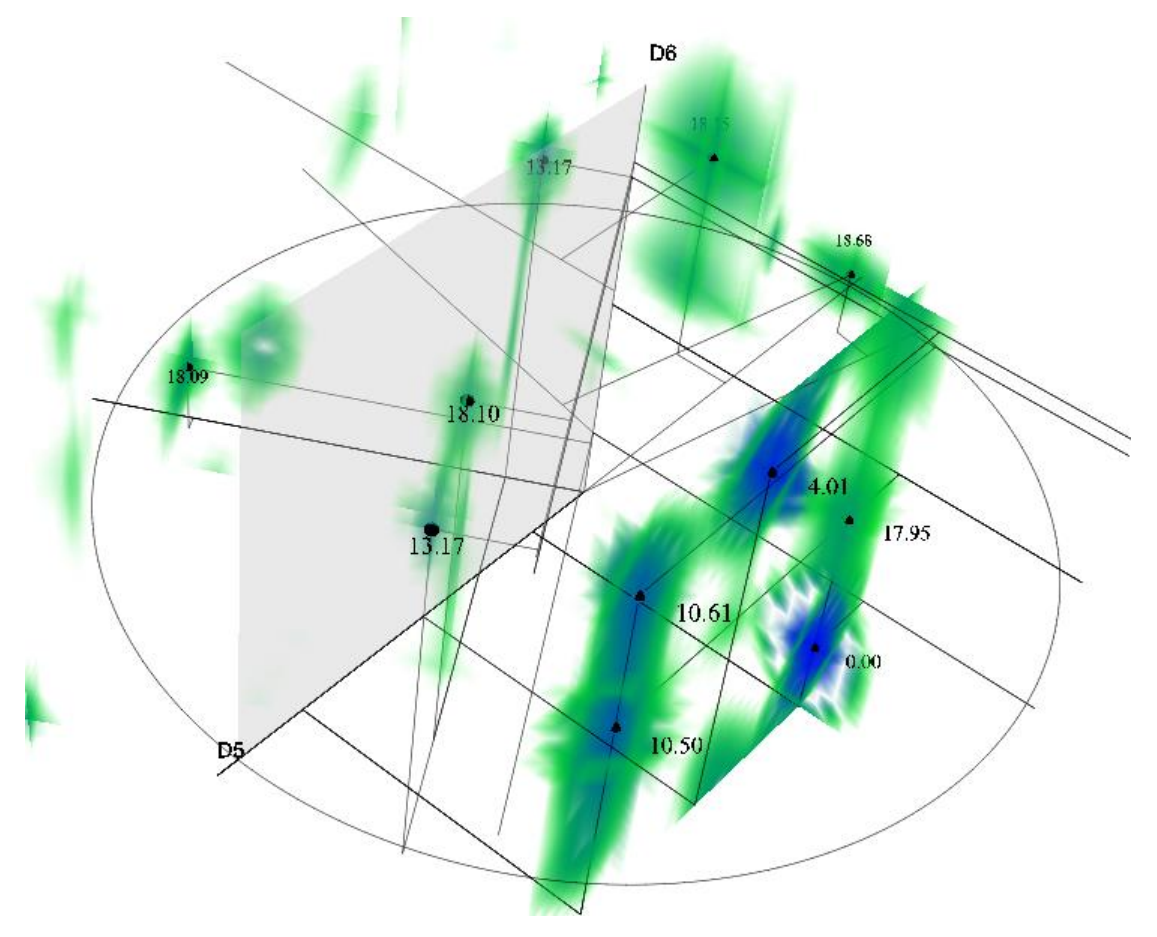

Figure 3. Part of 5D PES with the first 11 minima for glyceraldehyde

\section{SOME EXAMPLES FOR COMPLEXE CONFORMATIONAL LANDSCAPE OF MOLECULES}

We investigated conformational landscapes of a number of molecules by the developed postprocessor. Glyceraldehyde and fumaric acid with four and succinic acid even with five axes of internal rotation were predicted to exist in the form of several conformers.
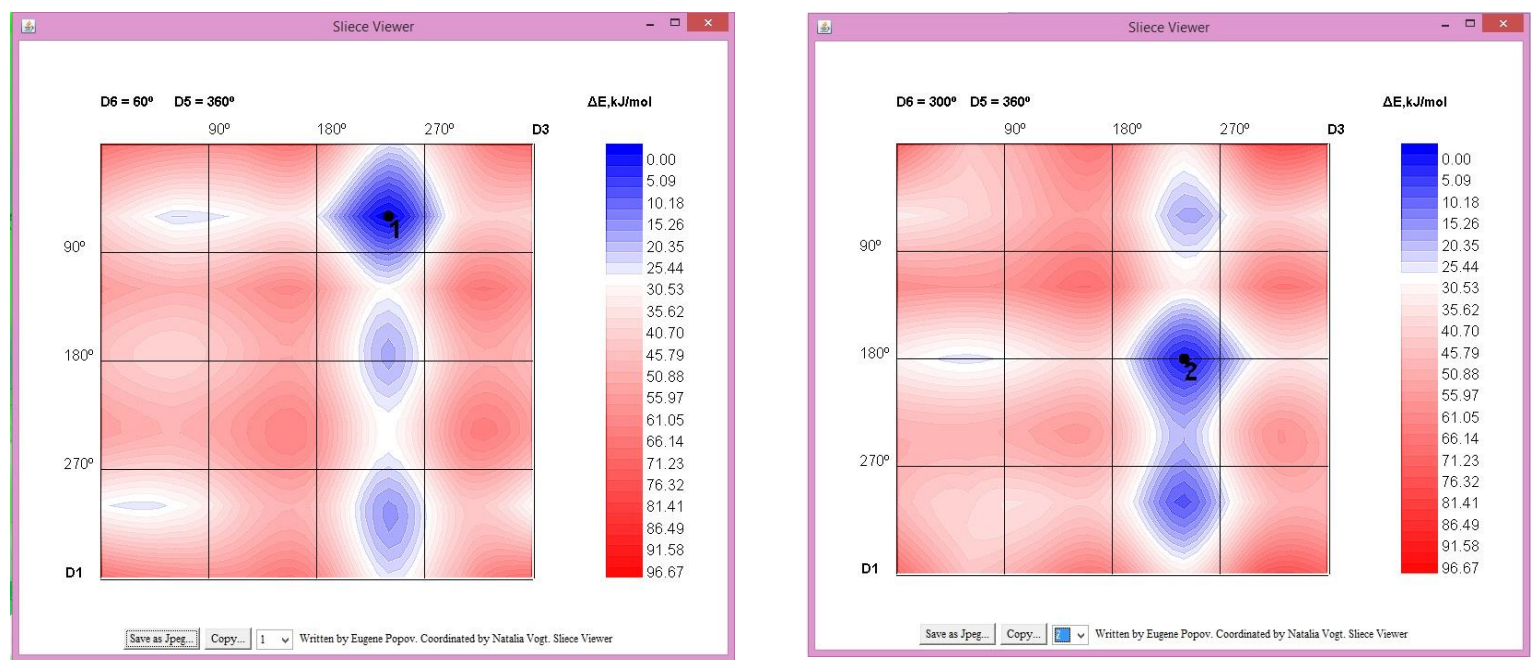

Figure 4. PES slices for 2 most stable conformers of glyceraldehyde 
Finding the stable conformers of several molecules in the gas phase was carried out in several experimental and computational studies (Vogt et al, 2007 - 2011). The developed post-processor was benchmarked by results of these studies and applied for finding stable conformations (Vogt et al, 2009). Its application helped to find 36 and 18 stable conformations for glyceraldehyde (Vogt, 2009) and succinic acid (Vogt, 2011), respectively. The post-processor permits automating the analysis of a multidimensional energy hypersurface and it looks as a systematic and reliable tool for conformer finding. Figure 4 demonstrates the sample slices of energy hypersurface (see hypersurface in Figure 3) through two stable conformers of glyceraldehyde.

\section{CONCLUSION}

The multidimensional postprocessor is aimed at processing potential energy hypersurface calculated by GAUSSIAN program. It also provides a systematic analysis of multidimensional energy hypersurfaces for minima finding. The solution of this problem is important for the study of the complex conformational landscape (for instance of biomolecules). The grid condensation technology significantly improves the accuracy of the PES minima finding. Besides, the post-processor has a very attractive visualization platform that allows the user to analyze the complete conformational landscape. In addition, it seems promising to use the postprocessor for solving other problems, for example, for searching the lowest-energy path between the local minima of multidimensional hypersurface, i.e. for finding the way of the interconversion between the conformers. This purpose can be challenge for the further research.

\section{ACKNOWLEDGEMENT}

The work was supported by the Dr. B. Mez-Starck Foundation (Germany).

\section{REFERENCES}

Borg I. et al, 2010. Modern Multidimensional Scaling Theory and Applications. Springer Series in Statistics. Springer, 2nd edition.

Etemadpour R. et al, (2016). Choosing Visualization Techniques for Multidimensional Data Projection Tasks: A Guideline with Examples. 166-186 pp 598. 166-186. 10.1007/978-3-319-29971-6_9.

Flanagan, M. T. 2014. Java Scientific Library http://www.ee.ucl.ac.uk/ mflanaga/java/index.html.

Foley J. D. et al, 1990. Computer Graphics: Principles and Practice. Addison-Wesley. ISBN 978-0-201-12110-0

Frisch M.J. et al, 2003. GAUSSIAN 03, Revision E.01. - Pittsburgh PA : GAUSSIAN , Inc., Wallingford.

Gotler S. et al, 1996. The Lumigraph. In Computer Graphics, Annual Conference Series (Proc. SIGGRAPH '96):43-54.

Jollife I. T., 1986. Pincipal Component Analysis. Springer-Verlag.

Kohonen T., 2001, Self-Organizing Maps, Third edition, Berlin: Springer-Verlag.

Niemeyer P. et al, 2013. Learning Java, 4th Edition. A Bestselling Hands-On Java Tutorial. O’Reilly Media, Inc.

Selman, D. 2003. Java3D. Programming. - Manning Publications Co Austin,- 302 p.

Squire R. 1997. Fundamentals of Radiology. 5th edition - Harvard, UniversityPress.

Tenembaum, J. B. et al, 2000. A global geometric framework for nonlinear dimensionality reduction. Science, 290:2319-2323.

Vogt N. et al, 2007. Molecular Structure and Conformational Composition of 1,3-Dihydroxyacetone Studied by Combined Analysis of Gas-Phase Electron Diffraction Data, Rotational Constants, and Results of Theoretical Calculations: Ideal Gas Thermodynamic Properties of 1,3-Dihydroxyacetone. Physical Chemistry A. - 2007. № 111. - P. 6434-6442. 2.

Vogt N. et al, 2009. Equilibrium structure and relative stability of D-glyceraldehyde conformers: gas-phase electron diffraction (GED) and quantum-chemical studies. Molecular Structure - The Netherlands, Elsevier, № 936. P. 125-131. 1.

Vogt N. et al, 2011. Determination of molecular structure of succinic acid in a very complex conformational landscape:Gas-phase electron diffraction (GED) and ab initio studies. Molecular Structure - The Netherlands, Elsevier, 2011. - № 996. - P. 120-127. 4.

Vogt N. et al, 2011. Molecular structure and stabilities of fumaric acid conformers: gas phase electron diffraction (GED) and quantum-chemical studies. Molecular Structure - The Netherlands, Elsevier, 2011. - № 987. - P. 199-205. 3. 